\title{
DA LETTERA APERTA A LE CERTEZZE DEL DUBBIO: LA VOCE AUTOBIOGRAFICA DI GOLIARDA SAPIENZA
}

FROM LETTERA APERTA TO LE CERTEZZE DEL DUBBIO: THE AUTOBIOGRAPHICAL VOICE OF GOLIARDA SAPIENZA

Viviana Rosaria Cinquemani

Universidad de Murcia

\section{Riassunto:}

L'obiettivo di questo studio è l'analisi degli scritti autobiografici di Goliarda Sapienza per comprendere il valore globale della sua produzione letteraria. Partendo da Lettera Aperta, dove la scrittrice si racconta sin dall'infanzia, ci soffermeremo sul racconto della malattia e la psicanalisi ne Il Filo di Mezzogiorno per approdare all'esperienza dentro e fuori dal carcere narrata ne L'Università di Rebibbia e Le certezze del dubbio.

\section{Parole chiave:}

Goliarda, autobiografia, psicanalisi, Rebibbia.

\section{Abstract:}

The aim of this study is to analyze the autobiographical writings of Goliarda Sapienza in order to understand the global value of her literary production. Departing from Lettera Aperta where she narrates her life from childhood, we will then deal with her illness and the psychoanalysis contained in Il Filo di Mezzogiorno to conclude with her experience inside and outside prison recounted in L'Università di Rebibbia and Le certezze del dubbio.

\section{KEY WORD:}

Goliarda, autobiography, psychoanalysis, Rebibbia. 


\section{INTRODUZIONE}

Una stele al cimitero di Gaeta la ricorda come “voce libera” (Providenti, 2010: 313), voce libera Goliarda Sapienza lo è stata nella vita come nella scrittura, basta accostarsi alla lettura dei suoi scritti per costatarlo. Purtroppo, è anche stata una voce dimenticata e a lungo trascurata, bisognava attendere la pubblicazione nel 2005 de L'arte della gioia in terra straniera (in Francia e in Germania) per far soffermare su di lei l'attenzione della critica italiana e dei lettori, e averne come effetto la conseguente pubblicazione, nel 2008, anche nella patria natale. Ma Goliarda Sapienza, come scrittrice, esiste ancor prima de L'arte della gioia, in queste poche pagine ci occuperemo, infatti, dei suoi scritti cronologicamente anteriori o paralleli, alla stesura e alla 'limatura' del suo capolavoro, mi riferisco al suo ciclo autobiografico ${ }^{1}$, a cui appartengono Lettera Aperta Il filo di mezzogiorno, L'Università di Rebibbia, e Le certezze del dubbio, con l'obiettivo di contribuire a recuperare e a capire oggi il messaggio della sua produzione, forse meno nota, e soprattutto a capire chi è Goliarda Sapienza come donna, come femmina, e come scrittrice, e per addentrarci nel suo microcosmo vitale poliedrico e autentico.

Attraverso la lettura dei suoi scritti possiamo notare quanto sia la sua vita e la sua scrittura fossero lontane dalle convenzioni sociali dell'epoca, e non solo, e dalla 'norma', non perché lei se lo proponesse, ma perché la sua vita sin dall'inizio si sviluppa fuori da ogni schema, a cominciare dal nome che le viene dato in cui è racchiuso un mondo ancor prima di iniziare il proprio. "Goliardo" era il nome del figlio del padre di Goliarda, Peppino Sapienza, a cui era stato messo perché si trattava di un nome senza santi essendo lui ateo; per il padre essere 'goliardo' significava far stare insieme cose che altri considerano separate ed inconciliabili: lo spirito democratico e laico, la curiosità intellettuale, la passione mondana per il vino e le belle donne. (Providenti, 2010:66).Verso questo fratellastro Goliarda provò sempre grande pena pensando che fosse annegato per il peso del nome che portava convinta di questo forse perché i genitori scelsero di chiamarla con il diminutivo "Iuzza" (Providenti, 2010: 74, 85).

Goliarda, quindi, eredita il nome del fratellastro morto adolescente, assumendo il peso di quell'amore del padre verso il figlio che la morte gli aveva strappato, forse per permettere alla vita di rinascere dalla morte (Providenti, 2010: 77), metafora di un topico che ritroviamo nella vita e scrittura di Goliarda. La visione della vita del padre si sposa perfettamente con l'anticonformismo orgoglioso della madre, Maria Giudice ${ }^{2}$, socialista e sindacalista attiva.

Si tralasciano in questa sede gli scritti di natura autobiografica apparsi postumi. I titoli dei lib dell'autrice sui quali si centra l'articolo, si abbrevieranno rispettivamente con: LA, FM, UR, CD.

2 Esaustivo è il ritratto che ne fa Providenti in La porta è aperta.
In questi ultimi anni, sulla vita di Goliarda Sapienza abbiamo notizie sempre più certe, precise e dettagliate grazie principalmente alla biografia scritta da Giovanna Providenti nel 2010 e al lavoro di raccolta e sistemazione degli scritti realizzato dal suo secondo compagno e marito, Angelo Maria Pellegrino.

La vita di Goliarda è sempre stata presentata come straordinaria, un aggettivo che ci suggerisce una qualità estremamente positiva, ma leggendo i libri del ciclo autobiografico, vi possiamo anche scorgere dei lati poco piacevoli (i lutti che colpirono la famiglia, i tentativi di suicidio, i problemi economici....), per cui preferirei utilizzare l'aggettivo di 'insolita'. Ricordiamone qualche elemento fondamentale che ha inciso sulla sua formazione come donna e come scrittrice: i genitori entrambi vedovi con figli che istaurano una relazione senza crismi religiosi, essendo entrambi laici, oltre che anarchici e antifascisti, il rapporto con i fratellastri e le sorellastre vissuto liberamente secondo vincoli affettivi e non di sangue, i rapporti col vicinato del suo quartiere che le hanno permesso di avere contatti con tutti i ceti sociali e soprattutto con 'vinti', essendo il padre avvocato dedito alla difesa dei più poveri, i suoi primi passi all'interno dell'Accademia d'Arte Drammatica a Roma, la fondazione, insieme agli amici, del "T45", una compagnia teatrale d'avanguardia, l'entrata nella Compagnia del piccolo teatro d'Arte che porta in scena i lavori di autori proibiti dalla censura fascista, il compagno Citto Maselli con cui condivide il suo talento e il suo mestiere da “cinematografara" (Providenti, 2010: 201), la partecipazione in alcuni film, i tentativi di togliersi la vita, gli elettroshock per curare la depressione, e infine l'approdo alla scrittura. ${ }^{3}$

Nella vita e nella professione, Goliarda si è sempre distinta, nel bene e nel male, ma senza la voglia, la pretesa o l'ambizione di farlo. Goliarda ha avuto sempre il coraggio di esprimere se stessa e nel farlo non è mai rientrata in nessuna definizione né si è autoproclamata seguace di qualche scuola.

Nel 2012 con la volontà di renderle omaggio, la scrittrice Pina Mandolfo ${ }^{4}$ ha curato un documentario, L'antigattopardo. Catania racconta Goliarda Sapienza, diretto da Alessandro Aiello e Giuseppe Di Maio, un viaggio sentimentale che ripercorre i luoghi e le tappe della vita di Goliarda, e che coincide con una scoperta dei luoghi catanesi che ispirarono la nostra autrice, per restituire il ricordo di questa donna che ha definito come "un monumento alla libertà femminile".

Nella scelta di questa definizione per il titolo del documentario, probabilmente, c'è anche la rivendicazione di non volerla associare al protagonista del romanzo di Tomasi di Lampedusa, a cui l'aveva accostata, seppur da una prospettiva diversa,

3 Le notizie biografiche sono tratte da Providenti (2010) e dai libri analizzati.

4 Ringrazio Pina Mandolfo, scrittrice e co-fondatrice della Società delle Letterate, per avermi messo a disposizione il documentario in formato $\mathrm{dvd}$. 
Adele Cambria (2006) in un suo articolo. La giornalista (amica di Goliarda) ne voleva mettere in luce la grandezza e lo stesso destino comune che ne vede il successo postumo, presentando il personaggio di Modesta, come "la nuova Gattoparda", una lettura delle intenzioni di questa giornalista spesso erroneamente fraintesa e attaccata con sarcasmo.

Anti-gattopardo, al contrario, è una definizione che ben descrive la figura di Goliarda Sapienza, in cui il vissuto e la scrittura sono agli antipodi dell'accettazione gattopardiana della vita; perché in Goliarda c'è sempre una voglia continua di rinnovarsi, di rinascita così lei legge e analizza il suo tentativo di suicidio, non affinché tutto rimanga com’è, non per salvaguardare un ordine precostituito, ma al contrario per minarlo, azzerare tutto e ricostruire una nuova Goliarda. Ne Il filo di mezzogiorno, attraverso le parole del medico, leggiamo: "Ci sono suicidi veri e suicidi, come è stato il suo, che non sono altro che un'azione vitale, un gesto per uscire fuori da una morte lenta, da una situazione difficile. Cerchi di ricordare: lei non voleva morire, voleva solo cambiare" (p. 59).

Questo non significa che in lei troviamo la perfezione, al contrario, nella sua vita ritroviamo anche delle contraddizioni o contrasti che la stessa autrice ci fa notare, quasi a ricordarci la sua natura umana, e che in ogni cosa esiste anche l'opposto, lei stessa appunta sui suoi Taccuini in data 22/1/1990 che il sottotitolo dei suoi romanzi dovrebbe essere Autobiografia delle contraddizioni (Providenti, 2010: 308).

Goliarda Sapienza non è una scrittrice che possiamo definire appartenente a una scuola, a una corrente, come la stessa afferma, "non appartiene a nessuna chiesa" (Providenti, 2010: 82). Ad una lettura superficiale la si associa facilmente all'ambito femminista, ma anche se ne condivide degli aspetti o anche se la sua vita in certe circostanze ci ricorda rivendicazioni femministe, non ne ha mai fatto bandiera, sarebbe riduttivo voler ridimensionare la sua voce e fissare un obiettivo politico e sociale alla sua scrittura. Così leggiamo in Providenti (2010: 80): "Goliarda non partecipa al movimento femminista di questi anni. Collettivi ed associazioni le piacciono poco: le sembrano un modo come un altro per evitare di assumersi responsabilità personali".

Nei suoi scritti gli equilibri fragili e imperfetti di una persona e di una donna e il suo continuo oscillare tra momenti di entusiasmo e momenti di depressione, non sono mai stati letti come disturbo mentale permanente, né quando tenta il suicidio, né quando, reduce dalla terapia basata sugli elettroshock, ha problemi mnemonici. Tuttavia, viene sempre notata come una voce fuori dal coro, forse fu questo il motivo per cui un medico siciliano decide di sottrarla all'elettroshock, molto in voga negli ambienti medici italiani dell'epoca, e la prende in cura: “Come mai gli baciavo le mani? "Lo sa signora che anche quando la venni a trovare in clinica mi prese le mani in questo modo? Il suo gesto mi spinse ad occuparmi di lei. Questo è un gesto di chi vuol vivere" (FM, p. 59). Oppure come avviene in carcere dove tutti, dopo la diffidenza iniziale e avendola conosciuta si contendono la sua compagnia e sviluppano nei suoi confronti un atteggiamento protettivo (UR, p. 136), come leggiamo nelle parole di una compagna che si accinge a uscire dal carcere "Tu se' stata un amore a prima vista per me, te lo devo di'... [...] Addio signo', e tu Roberta, tienimela bene perché è pura e santa 'sta creatura...Finché c'ero io, come ho detto a tutti, finché c'è Annunciazione qua, la signora nun deve temere niente da nessuno, ma adesso vado via... Me la guardi eh, Robbe'."

La sua scrittura è autoliberante, è terapeutica, curativa, ma è soprattutto una testimonianza umana per sfidare la morte e l'oblio e per allungare di qualche momento la vita su questo pianeta. ${ }^{5}$

\section{LeTtERA APERTA: RACCONTO DI UNA VITA A NUDO}

Lettera aperta rappresenta il suo esordio letterario ufficiale, finito di scrivere nel 1963 e pubblicato nel 1967. “Non è per importunarvi con una nuova storia né per fare esercizio di calligrafia, come ho fatto anch'io per lungo tempo; né per bisogno di verità - non mi interessa affatto,- che mi decido a parlarvi di quello che non avendo capito, mi pesa da quant'anni sulle spalle" $(L A, 15)$. Si apre con queste parole il primo romanzo di Goliarda Sapienza, in cui si rivolge ai lettori, che contemporaneamente saranno specchio di se stessa. Siamo di fronte a un diario segreto aperto e diretto. Goliarda inizia a scrivere, credendo nel potere curativo della scrittura, e lo fa senza fronzoli mettendosi a nudo, non obbedendo totalmente al principio di verità, ma senza censure e senza moralismi: "Ma, visto che questa ricerca solitaria mi portava alla morte - sono stata due volte per morire "di mia propria mano", come si dice - ho pensato che sfogarsi con qualcuno sarebbe stato meglio, se non per gli altri almeno per me" (Ibidem). In questo libro-diario, Goliarda racconta e mescola la sua infanzia e il suo presente, vissuti fuori dalle convenzioni sociali; un contenuto 'avanzato' o forse troppo spregiudicato per il contesto storico, sociale e culturale in cui viene pubblicato il libro, siamo in Italia, nel $1967^{6}$. A questo proposito Pellegrino (FM, 10), afferma:

Oggi, dopo quasi quarant'anni, è difficile capire cosa volesse dire per la cultura italiana di metà anni Sessanta scrivere due romanzi come Lettera Aperta e Il filo di mezzogiorno. Bisogna pensare a che cos'erano quegli anni prima dell'esplosione del '68, con cui cominciò a cambiare tutto. Se prendiamo in mano qualsiasi opera di narrativa di quel periodo, non troveremo niente di simile in quanto a originalità, ricchezza di stile e di visione, senso alto della letteratura: della tradizione insieme all'innovazione.

$5 \mathrm{Il}$ concetto racchiuso in questa frase lo leggiamo ne Le certezze del dubbio (166).

6 Nel 1969, il regista Citto Maselli, compagno per quasi un ventennio di Goliarda Sapienza, ne trae ispirazione per un film Lettera aperta a un giornale della sera, in cui Goliarda interpreta se stessa: un'intellettuale indaffarata e fuori dal coro (Providenti, 2010: 17). 
E come sottolinea Hernández (2009: 143), sempre riferendosi al contesto culturale in cui scrive Goliarda (anche se a proposito de L'arte della Gioia):

Puede decirse que en el panorama narrativo de aquel momento, después de los experimentalismos de neorrealismo y del objetivismo, una propuesta como la de Sapienza, que pretendía escribir una vasta novela popular destinada a las mujeres, como había hecho Elsa Morante con La Storia no entraba en ninguna etiqueta como habia her estilo, calificado de espontáneo, descuidado, improvisado.

Nell'introduzione alla terza edizione del libro del 2012, Dacia Maraini, afferma che "Lettera Aperta è il suo libro più compiuto, certamente il più significativo" (11) Forse perché ci presenta, per la prima volta, approfondendo o solo sfiorandole tutte le vicende della sua vita che il lettore, chiamato in causa in una cooperazione attiva, riordina per avere il ritratto globale di questa scrittrice e, a sua volta, funge da pretestostimolo per aiutarla a riordinare la sua vita:

"Ho bisogno di voi per sbarazzarmi di tutte le cose brutte che ci sono qui dentro" (16) come una doppia catarsi: fisica 'dentro' la stanza, e spirituale 'dentro' il suo mondo interiore.

Tra i ricordi raccontati, i più significativi per capire questa autrice credo che siano: la figura dei genitori, in primis, l'educazione e l'istruzione ricevuta, il suo credo, la sessualità, i rapporti interpersonali.

La madre di Goliarda in quest'opera, appare come una donna che si distingue da tutte le altre donne, a sottolineare la sua straordinarietà le parole dello zio (fratello del padre) che quando la nominava si riferiva a lei come "la incomparabile donna Maria, intelligente più di un uomo" (39). L'accostamento della madre alla figura dell'uomo che la priva della debolezza considerata caratteristica femminile, porta Goliarda a considerare negativamente l'arrivo del ciclo mestruale che fa di una bambina una "donnetta", la cui unica aspirazione è quella di sposarsi e mettere al mondo dei figli, così come 'vigeva' nell'ordine sociale e religioso dell'epoca: "Sono donnette che non sanno fare altro che aspettare un marito e anche aveva detto 'Tu Goliarda non sei una donnetta'. Io infatti non volevo un marito, ma un compagno come lei... Certo, non veniva a tutte, ma solo alle donnette, e a me non sarebbe venuto. Sarei stata come mia madre. Avrei parlato come lei con gli uomini, e se non aspettavo un marito il sangue non mi sarebbe venuto" (110).

Dall'altro lato, il padre che le ricorda di non poter sfuggire al suo destino di 'signorina', che esige determinati comportamenti, e la avverte: "Senti, Goliarda. Ho saputo. Non vorrei che ti scordassi che siamo in Sicilia. Non vorrei che pensassi che anche se ti lasciamo la libertà di andare e... Sei una signorina oramai. Devi sapere che è pericoloso, gli uomini cercano sempre il loro piacere" (118). Il suo amore da bambina verso il padre passa, contemporaneamente al suo passaggio verso l'età adulta, a una dimensione di distanziamento che si evince nel libro nell'uso dell'appellativo 'l'avvocato' quando si riferisce a lui.

Goliarda ci racconta anche il suo peculiare rapporto con l'educazione ricevuta e l'istruzione che le viene impartita da un precettore, il professore Jsaya, e che in larga parte fu anche autodidatta, sebbene frequenti per un breve periodo la scuola, che lascerà anche con l'appoggio, da parte del padre. Ricorda l'autrice che un giorno il padre vedendola tornare da scuola con il grembiule le chiese: "che fai con quella maschera schifosa?" (150), attraverso quel simbolo il padre aveva attaccato l'istituzione, Goliarda andò in terrazza, lo bruciò e non tornò più a scuola, anche perché "studiare non potevo mi veniva sonno" ma in compenso "leggevo e imparavo a memoria tutti i lavori teatrali che trovava per casa" (151). Un altro ricordo significativo risale a quando Goliarda disse al suo compagno di banco, mentre entrava in classe l'insegnante di religione, che stava arrivando la siringa con la quale s'iniettava la droga della religione, il compagnetto fece la spia e Goliarda venne punita e cacciata dalla classe, successivamente il padre la fece esonerare (64).

Goliarda credeva in un panteismo spinoziano in cui tutto è divino, ma per tagliare corto diceva di essere atea e materialista; ${ }^{7}$ anche se nel corso della sua vita, da piccola, aveva mostrato curiosità verso la religione cattolica e i suoi simboli, una curiosità che non era diventata altro, a casa non avevano ostacolato la sua curiosità, ma regnava un clima laico, lo vediamo attraverso la reazione alle parole del fratello che la rimprovera per aver mentito sul suo nome e aver detto ad alcuni bambini di chiamarsi Maria: "A casa mia scusarsi era ritenuto un gesto dolciastro e cattolico" (46), "Non si chiede scusa chiedere scusa è il sistema che usano le donnette per rifare i loro sporchi peccatucci. Sei un individuo, e sei responsabile delle tue azioni. Non chiedere scusa, ma cerca di riparare" (47)

Questi aspetti della sua vita la portano a uno scardinamento della visione convenzionale e spesso manichea della realtà, portando a tollerare, ad accettare e a giustificare persino l'incesto tra una madre e il figlio, cosa socialmente condannata come abominevole: "penserò ai vostri abbracci come a un terribile fatto di natura, come la nascita e la morte" (132).

Se già i fatti narrati in Lettera Aperta ci mostrano un vissuto inusuale, ancor più lontano dal comune è il modo e lo stile in cui si svolge la narrazione. Seppur con maggiore leggibilità, lo stile ricorda, lo stream of consciousness di Joyce, in cui i ricordi vengono presentati così come affiorano nella memoria, apparentemente senza ordine,

7 Al riguardo, si consulti la testimonianza di Angelo Pellegrino, intervista disponibile su http://www. youthlessfanzine.com/goliarda-sapienza-dallarte-della-gioia-alluniversita-di-rebibbia 
che lei si preoccupa però di sistemare. S'intersecano i piani narrativi tra passato e presente, nel ricordo le voci si confondono e nel presente si ordina: "Il professor Isaya diceva: 'una persona sola è la solitudine, l'impotenza. In due si fa già un sindacato' No, questo non lo diceva il professor Isaya, lo diceva mia madre, era lei la sindacalista." (31).

Curioso l'avvertimento al lettore nel capitolo 7, in cui scrive a inizio pagina e tra parentesi "Si può non leggere", quasi come una carezza per non tediarlo, o al contrario come uno stratagemma per stuzzicare la sua curiosità, e la nota finale in cui si giustifica di aver raccontato per non dimenticare. Si tratta del capitolo in cui ci parla di Ercole (padre del suo compagno) e della sua morte, verso il quale nutre una profonda stima, ammirazione, amore e gratitudine: "Ed è per questo che lo ringrazio oggi di avermi liberata e fatto ricordare come si muore. Si muore per lasciare il meglio di sé a quelli che ti hanno saputo leggere" (35).

\section{Il filo di mezzogiorno: il labile confine tra la vita e la morte}

Se in Lettera aperta il potere della scrittura rappresenta uno sfogo liberatorio per fare ordine, ne Il filo di mezzogiorno la scrittura assume totalmente un potere terapeutico per ricostruire i tasselli di una vita passata e presente, sconvolta dagli effetti che l'elettroshock ha prodotto sulla sua memoria. Si tratta di un libro in cui la scrittrice riprende a raccontarci la sua vita alla luce di quanto le è successo dopo il secondo tentativo di suicidio e questa volta non per mettere ordine, ma per recuperare i ricordi e per guarire.

I fatti narrati si ricollegano come con filo invisibile a quelli raccontati nel romanzo precedente, il titolo stesso lo ritroviamo tra le pagine di Lettera Aperta in cui l'amicasorellastra, Nica, in uno dei suoi racconti di saggezza popolare avverte: "Non andare tra le viti nel filo di mezzogiorno: è l'ora che i corpi dei defunti, svuotati dalla carne, con la pelle fina come la cartavelina, appaiono tra la lava" (121). E proprio in questo libro ci ritroviamo a tratti in una dimensione rulfiana in cui i morti convivono con i vivi, in cui le loro voci messe sullo stesso piano narrativo si confondono e si sovrappongono.

La peculiarità di questo libro non è solo il contenuto, ampliamente riconosciuto come primo esempio di stesura di processo di psicanalisi realizzato dal paziente, ma anche il modo in cui viene presentato questo processo attraverso la narrazioni di fatti, di sentimenti, di stati d'animo.

Ne Il filo di mezzogiorno la sua scrittura ci ricorda lo stile di Richardson il writing to the moment e si avvicina di più allo stream of consciusness di Joyce, cioè la narratrice recupera a distanza di tempo attraverso la forma autobiografica, un'esperienza del passato, che però viene raccontata e descritta contemporaneamente al fatto narrato, come se fosse 'a caldo', dove il presente della narrazione si fonde e si confonde con il piano narrativo dell'analessi.

Rispetto a Lettera Aperta, cambia la punteggiatura, sono più frequenti i puntini sospensivi, che indicano la struttura aperta delle frasi e quindi una scrittura in movimento come i pensieri, e diminuiscono i periodi definiti e chiusi dal punto.

Il contenuto è anticonvenzionale, leggiamo che il rapporto che s'instaura tra Goliarda e il suo medico non è quello gerarchico della subordinazione del paziente ammalato al medico guaritore, ma si dipana in una dimensione simbiotica che alla fine capovolgerà i ruoli, Goliarda guarisce e il medico si ammala. In un primo momento il medico si apre e confessa le sue sensazioni "Lo sa che da quando abbiamo cominciato a lavorare insieme, nei miei sogni, e anche da sveglio, stanno affiorando cose nuove? Un analista deve sempre continuare l'auto-analisi. Se lei mi aiuterà, io la guarirò e lei guarirà me. Questa mattina mi sono ricordato un canto dei pescatori delle isole Eolie che non ricordavo neppure di sapere" (74).

Il medico riconosce, inoltre, in Goliarda delle qualità eccezionali: "[...] sa che mi accorgo di tutto quello che le avviene. Sì, è vero, ma questo è perché lei ha una sensibilità straordinaria e una possibilità di associazione e sintesi delle espressioni negli individui. Lo sa che lei avrebbe dovuto fare il medico? Ed è appunto per questo che lei è una paziente particolarmente difficile ed interessante. Io solo posso guarirla"(87).

Tuttavia, alla fine, lo stesso medico, probabilmente per timore di ciò che si sta producendo, si ritrae infastidito, cerca di ristabilire i ruoli:

$[\ldots]$ [Che fa lì impalata?»

«Ma dottore, cosa è successo?»

«Non cominciamo con le domande, cosa vuole che sia successo. Non è successo niente. La finisca di occuparsi di me, sono io che debbo occuparmi di lei. Lei è la paziente. La finisca!» (163)

In questo processo di guarigione Goliarda s'innamora del suo medicoeglielo dichiara, fino al punto di confessargli di desiderare un figlio da lui, il medico ridimensiona e sminuisce il suo sentimento definendolo soltanto un semplice transfert. "«No dottore, il fatto è che non ce la faccio più...ho aspettato che questo trasporto che sento per lei, questo transfert, come lei lo chiama, passasse, ma è passato quasi un anno e... lei mi aveva detto che in tre o quattro mesi si sarebbe risolto in sede psicanalitica e...» «Si fidi di me signora, vedrà che dipaneremo questa matassa imbrogliata di sentimenti ed emozioni confuse che lei crede che sia amore. L'amore non esiste. Si fidi di me... »"(139-140)

Goliarda in questa fase è una persona che si questiona la norma, s'interroga e interroga il suo medico su cosa s'intende per 'normalità' a proposito dell'amore, ma il concetto che esprime va anche oltre l'amore, in una dimensione vitale e sociale generale: 
La sua mancanza di aggressività è la sua malattia. Lei deve lasciare che le unghie le crescano, deve imparare a lottare, imparare a farsi valere. Dice di essere innamorata di me, ma non una parola, non una domanda su mia moglie, non una protesta. Questo non è naturale, non è genuino, è artefatto, non è naturale." "Perché secondo lei viviamo secondo ( perché dovri uccider gli alti Averla tutta per me? Mi scusi, ma proprio questo atteggiamento possessivo che lei mi vuol far passare per genuino, sano, mi pare malato e ed immorale. E poi le parole genuino, naturale, non mi piacciono, io amo così e sentendo che anche lei mi ama... (149).

Qui traspare il suo concetto d'amore non come possessione esclusiva ed egoista dell'altra persona sia essa uomo o donna, ne sono una testimonianza le donne condivise con Citto Maselli nei loro singolari ménage à trois altalenanti tra l'amicizia e l'amore, tra la passione e spirito materno. L'amore vissuto così come sgorga dal cuore, senza condizionamenti, spontaneo e che essendo 'umano' prima che sociale, rispetta i ritmi delle emozioni e della vita (anche se poi con Angelo Pellegrino sceglierà di sposarsi):

[...]...come mi aveva raccomandato mia madre, non facemmo la sciocchezza di sposarci o solo di pensarci, facemmo il patto di stare insieme così, fino a quando la gioia di stare insieme sarebbe durata... ed eccoci, a sedici anni... sembra impossibile..."

"Ah, fu sua madre ad imporle questo?" "Impormi? Ma che dice? Era giusto, perché fare contratti disumani e bestiali?" “Ed è così sicura che sia stato un bene? Lei è una donna, signora, e forse si sarebbe sentita più protetta.." "Ma che dice? A volte mi sembra un piccolo conformista, un... (103).

Il processo di cura con questo psichiatra di origini messinesi, Ignazio Majore, inizia nel 1962 e si protrae per 3 anni, sono anni che scardinano le certezze di Goliarda in cui lei torna indietro per andare avanti: "dovevo ritrovare mio padre, mia madre, Nunzio, Nica, i miei fratelli, le mie sorelle, dovevo ritrovarli e ricostruirli in una vita loro" (176).

E mentre Goliarda ricostruisce la sua memoria, il medico le spiega la sua tendenza patologica: "Sa come si chiama il meccanismo di riesumare nel presente situazion passate per ritrovare quelle emozioni, negative o positive che siano, perché solo quelle si sono conosciute e sperimentate e si identificano quindi con la vita stessa? Destino coatto. Era giustissimo quello che diceva: destino coatto, sì." (82). E le diagnostica la sindrome abbandonica:

Vede signora, il bambino ha bisogno di affetto come di cibo, e quando questo viene a mancare si hanno le forme di depressione che lei ha: che altro non sono che una svalutazione di se stessi, un sentirsi abbandonata dagli altri che non è altro che il riproporsi lo stato di abbandono rimosso, subito apparentemente nell'infanzia. Sa come si chiama questo tipo di nevrosi? Abbandonica (76)
Ma io non l'abbandono, né voglio che cambi perché mi piace così com’è: voglio solo che si rinforzi un tantino, che prenda coscienza di sé, prenda coscienza che lei è una donna di valore che nessuno vorrebbe mai abbandonare. Si deve rendere cosciente, deve portare alla coscienza il meccanismo abbandonico che la fa troppo paziente e paurosa con gli altri. Lei non ha bisogna degli altri, o almeno non cosi paziente e pas cos come nelle fantasie infantili si è raffigurata (81).

Secondo il medico, Goliarda doveva non solo recuperare i ricordi delle persone del suo passato, ma inserirli nella loro giusta dimensione: “ $« i$ morti ingigantiscono nelle nostre emozioni, nel ricordo, e non si possono contraddire, e nell'impossibilità d contraddirli, loro prendono possesso di noi più di quanto non fosse stato da vivi. [...] I morti sono morti e noi siamo vivi».

Come ci fa notare Providenti (2010: 279):

Il percorso terapeutico, indagato a fondo nella sua anima, l'aiuta a perdonarsi de molti sensi di colpa che prova nei confronti dei propri genitori, ed in particolare a casa della malattia della madre. Mentre dialoga con il suo analista si mette in gioco interamente, i dolori infantili, i meccanismi di difesa e le paure della sua vita, imparando ad accettarsi di più. Man mano che la consapevolezza di se stessa e dei propri meccanismi "coatti" aumenta, Iuzza inizia anche a lasciarsi andare a una scrittura meno preoccupata degli esiti narrativi e più schietta.

In questa fase della vita di Goliarda, c'è anche un allontanamento da Citto, il suo compagno, che a sua volta sceglie di entrare in analisi con lo stesso medico. Non c'è nessun dramma in quest'allontanamento che invece è vissuto con la naturalezza di un rapporto che si trasforma, come tutte le cose umane... Ma se Goliarda guarisce, risolve e si libera dai sensi di colpa, l'analisi di Citto è bruscamente interrotta dalla sopraggiunta 'follia' del tanto rinomato medico, una follia che in fondo è anche una liberazione dal suo ruolo, da ciò che rappresenta e dalle certezze costruite su cui poggiava la sua vita personale e professionale. Nel racconto di Citto a Goliarda lo vediamo passivo, impassibile, scettico e con l'unica certezza dell'inutilità della sua professione:

Mah, cose da non credersi, inimmaginabili, ancora non ci credo. Il dottore ha lasciato la sua professione, non crede più nella psicanalisi...Hai capito? Va dicendo che non esiste neanche la psicologia, puoi immaginare! Sono un po' sbalestrato. [...] Sta in casa barricato e quando arrabbiato, puoi capire, ho insistito, mi ha fatto la grazia di vedermi e mi ha liquidato con quattro parole che non stanno né in cielo né in terra. Naturalmente gli ho detto quello che pensavo, ma non si è scomposto e quando gli ho chiesto il nome di un altro medico mi ha dato il nome di... un giovane freudiano ortodosso dicendo: Non è di nessuna utilità, ma se proprio insiste...

Anche Goliarda in attesa di risposte va a trovare il medico, che però le sbatte la porta in faccia. Proprio colui che aveva identificato in Goliarda la sindrome abbandonica, che le aveva promesso che non l'avrebbe abbandonata, adesso la abbandona, interrompe 
l'analisi senza voler più sapere niente della sua professione. In questa solitudine Goliarda non si perde, la accetta, la esplora e ne rinasce rinnovata, come ricorda Arena (2012: 155): "la scrittura è il segreto che Goliarda scopre e sceglie come destino, qui la scrittrice nasce e muore senza fine".

\section{L'università di Rebibbia e le certezze del dubbio: dentro e fuori dal carcere}

Questi due romanzi autobiografici ${ }^{8}$ rappresentano due momenti di grande importanza nella vita di Goliarda Sapienza, il primo è l'entrata in carcere per il furto di alcuni gioielli a un'amica e il secondo momento è l'uscita dal carcere. Due momenti che la segnano e la cambiano come persona e come scrittrice: "per la legge dell'uomo un tuo modo di essere è stato cassato, la fedina penale macchiata, le mani insozzate dall'inchiostro per le impronte digitali: quella che eri prima è morta civilmente per sempre."

Per chiarire e capire cosa effettivamente rappresentò per Goliarda l'esperienza de carcere, leggiamo le considerazioni che la stessa scrive nei suoi Taccuini in data 1989 (Providenti, 2010: 291):

\begin{abstract}
L'entusiasmo che provai appena rinchiusa a Rebibbia mi dice molte cose: l'entusiasmo di cui ho detto si manifestava - specialmente nei tre giorni di cella $\mathrm{d}^{\prime}$ isolamento- in una pace e soddisfazione etica indicibile, come qualcuno che finalmente- abbia rimesso piede nel suo paese d'origine da cui un evento nemico l'aveva sradicato. Questo paese era quello dei poveri, umiliati e offesi, di cui mia madre mi illustrava le storie (del brigante Musolino, i soggiorni di lei in carcere, ecc.) le tante magnificenze, di solidarietà, apprendimento morale, studio di lotta di classe, ecc.
\end{abstract}

Già nel titolo è racchiusa la concezione positiva della sua esperienza interpersonale nel carcere romano di Rebibbia, che considera un "corso accelerato di vita [...] devo far fruttare ogni attimo di questo corso rapido di esperienza." (UR, 49), sebbene si tratti di un'esperienza che racchiude anche una forte critica al sistema penitenziario italiano. La sua carcerazione dura 5 giorni, ma lei li vive e li racconta come se fossero 5 mesi nell'ottobre del 1980, qui si ritrova tra i vinti, li aveva conosciuti a casa sua, nella sua città, ma qui li vive in modo privilegiato e totale.

Lo stile è diverso rispetto agli scritti precedenti, qui la sua scrittura ha un taglio più da documentario, è uno stile asciutto e visivo, ma preciso nelle descrizioni delle cose e dell'aspetto delle persone, siamo dinnanzi al tempo del carcere che ti fa osservare tutto nei dettagli: il cibo che ti viene dato, la cella in tutti i suoi angoli, la latrina, il sapone, la scopa... Tutti gli oggetti quotidiani e ordinari assumono protagonismo sotto la luce di

8 L'Università di Rebibbia edito da Rizzoli nel 1983, e Le certezze del dubbio edito da Pellicano Libri nel 1987. questo pezzo di mondo; la stessa cosa succede quando descrive i capelli, gli occhi, la stazza, la voce, i denti delle sue compagne...

Compagne che quasi per discrezione non descrive caratterialmente, ci penseranno loro nei loro interventi a fornire al lettore una chiara definizione:

Devo aver dormito a lungo perché quando apro gli occhi la finestrella dà più luce e dietro la porta, dopo tanto silenzio, si sentono scalpicci di passi affrettati, stridere di chiavi nelle toppe, rotolì di ruote metalliche. Scorgo allo spioncino un carrello immenso con due donne che ci trafficano intorno. Di una vedo solo la schiena enorme, l'altra, giovanissima e paffutella, i modi bruschi da contadina, mi porge un bicchiere di plastica una volta bianco ma oramai divenuto marrone dall'uso. $(U R, 9)$

Anche qui la narrazione è condotta in prima persona, ma non si rivolge solo a suo mondo interiore, coglie anche la dimensione dell'altro, mostra empatia verso un mondo diverso da quello finora conosciuto.

Anni fa, il suo intervento in una trasmissione condotta da Enzo Biagi ${ }^{9}$, produsse incredulità e perplessità nel giornalista che la ascoltava mentre lei parlava del carcere come di un microcosmo perfetto nella sua imperfezione, ma allo stesso tempo ne denunciava le condizioni in cui versava come luogo fisico, e ribadiva, inoltre, il concetto che le aveva trasmesso la madre, secondo cui il proprio Paese si conosce, conoscendo il carcere, l'ospedale e il manicomio. Una riflessione che aveva già espresso tra le pagine del suo libro: "Perché chi ha avuto la ventura di andarci, appena fuori, si vergogna e ne tace $\mathrm{o}$, chi non se ne vergogna, s'ostina a considerarla una sventura da dimenticare." $(U R, 47)$; “Fra noi larve di fuori si comincia perfino a sussurrare che le classi non esistono più. Poveri illusi! Cosa darei per trascinarli tutti - anche solo per una settimana- qui a Rebibbia a vedere la sintesi chiara e inappellabile del mondo di fuori col suo, ora per ora, eterno riprodursi del vinto e del vincitore, del servo e del padrone..." $(U R, 50)$

Senza fare un grande sforzo Goliarda riesce a farsi ben volere da tutti e a ricreare lì in un ambiente comunemente ostile, delle atmosfere familiari, benché all'inizio non sia facile:

Il mio modo di essere qui non va. Saranno i vestiti, anche se sporchi, di lusso qualsiasi donna capisce il taglio di un paio di pantaloni e di una camicia, il taglio dei capelli...O è solo il modo di muovermi? E poi ci sono le scarpe: non c'è niente da fare, si vede che sono scarpe da ottantamilalire. Tutto questo non lo posso cancellare, ma il mio modo di muovermi, di gestire sì...Come? Su questo è inutile teorizzare, la prossima volta devo capirlo da come mi guardano e agire di conseguenza $(U R, 15)$

9 Goliarda Sapienza intervistata da Enzo Biagi. Disponibile su https://www.youtube.com/ watch?v=ojXxjHr6MU0 
Con il carcere Goliarda sperimenta un'altra dimensione spaziale, la sua cella, e temporale, un tempo che all'improvviso si dilata, ma che è e deve essere un continuo presente: "«Quando uscirò»! Altra cosa da fare: non pensare mai al futuro in questo posto, il futuro è sempre pieno di porte immaginarie di cui credi di avere le chiavi, qui le chiavi le hanno inequivocabilmente gli altri" $(U R, 24)$

In carcere, istaura dei contatti che si trasformeranno poi in amicizie, come succede con Roberta o Marcella o Barbara, che saranno al centro del libro successivo.

Roberta è colei che le spiegherà questo strano sentimento che molti in carcere sviluppano: l'affezione carceraria, l'affezionarsi al carcere con il costante desiderio di volerci tornare anche dopo aver scontato la pena. La compagna le racconta i numerosi casi di persone che una volta fuori non riescono a riadattarsi e fanno di tutto per rientrare e recuperare l'equilibrio che lì avevano creato: "Non è possibile mantenere in piedi la costruzione ideale di noi stessi. Ritorna a vigere suprema la selezione naturale." (UR, 81)

L'inizio del libro successivo, Le certezze del dubbio, è concatenato alla fine de L'Università di Rebibbia, poiché racconta, diventandone testimonianza, l'affezione carceraria; l'autrice cioè, vuole ricreare quel mondo che aveva trovato in carcere, anche fuori da esso. La narrazione inizia con le riflessioni di Goliarda che viene "sbattuta fuori dal carcere" $(C D, 3)$, nel "grande carcere della libertà"(169). Un giorno per caso incontra in Tribunale, Roberta, la sua ex compagna di cella e con lei lentamente e non senza qualche difficoltà riallaccia i rapporti lasciati in sospeso in carcere. In loro notiamo la fatica di reinserirsi nel mondo esterno, perché non sono quelle di prima. Per non recidere il cordone con il loro 'passato', continuano a muoversi e a frequentare gente che ha sperimentato il carcere. Il ricordo del carcere quasi come un locus amoenus non tanto come luogo fisico, quanto come insieme di atmosfere.

Agli occhi di Goliarda, Roberta incarna, da una parte il suo riflesso nella difficoltà di reinserirsi, ed è anche l'unica persona che può capirla e alla quale confessa:

Non posso dirlo a nessuno, ma spesso la rimpiango Rebibbia. Temo proprio che avevi ragione quando scopristi che avevo gia qualche sintono dell'affezione al carcere... Come si chiama esattamente? - Lo sai benissimo. Sindrome carceraria. Perché la rifiuti? È dolce avere un rimpianto. [...] Ecco cosa avviene in quello stato che si chiama detenzione: la realtà, ricerca dei soldi, doveri, etiche, viene tagliata fuori ripotenziando al ma: lagliata

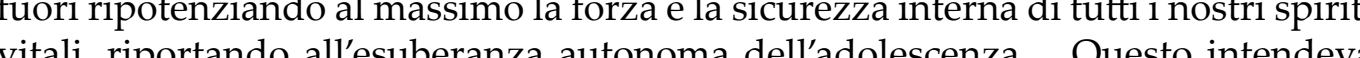
Stend i o Stendhal ogni qual volta riduceva il suo protagonista in una cella per metterlo in grado di ritrovare la sua integrità perduta." $(C D, 121)$

Dall'altro, Roberta fa scaturire in lei, il sentimento di maternità, per cui l'autrice proietterà su di lei la sua protezione e la sua preoccupazione. Roberta assumerà le sembianze della figlia mancata che cerca di partorire con gli strumenti messi a disposizione dalla scrittura: "«Perché scrivi Goliarda? - Per allungare di qualche attimo la vita delle persone che amo. - E con la loro anche la tua, eh, volpona?- Certo. Chi odia a tal punto la vita da non desiderare d'averla allungata almeno un po'?- Bene allora un giorno forse scriverai di me." $(C D, 166)$

Alla scrittura, come dicevamo in precedenza, affida il potere di allungare di qualche attimo la vita delle persone che ama. Scrittura come mezzo che ci permette di sopravvivere anche dopo la nostra morte fisica, scrittura come arte, che ci ricorda l'idea keatsiana ${ }^{10}$ di eternità dell'arte nei confronti della caducità della vita umana, affidare alla scrittura il compito dell'eternità

Sul piano linguistico ne Le certezze del dubbio, come ci fa notare Andrigo (2012:129), sono presenti due macro-livelli di differenziazione: una lingua scritta che caratterizza la narrazione e una lingua parlata che invece ritroviamo nei dialoghi. Goliarda trascrive il dialetto romanesco (riproducendone anche gli errori ) e delle forme gergali che alleggeriscono la narrazione e le conferiscono una maggiore vivacità, credibilità e realismo: "«Nun piagnevi ieri sera...Io pensavo che nun era la prima vorta... cioè nun ci avevi l'aria de 'na pivellina... E perché t’avrebbero da chiude' dopo?...Quanno arrivite chiudeno pe' impressionatte, fatte cuoce' ner tu brodo, così che al limite sbrocchi e parli»" $(C D, 28)$

\section{Considerazioni final}

I romanzi su cui ci siamo soffermati si caratterizzano per il tratto autobiografico, dove il soggetto narrante coincide con l'oggetto della narrazione, ma si tratta di romanzi tutti autobiografici in maniera diversa. Il risultato che ne deriva dalla lettura è il racconto di questa vita che si fonde e a tratti si confonde con l'arte della scrittura e della finzione.

La scrittrice non ci presenta la sua vita inserendola in un freddo ordine cronologico fatto di date e numeri, ma è un racconto spontaneo che il lettore legge e ricostruisce in progressione, non 'per bisogno di verità', ma quasi per renderle omaggio e realizzare il desiderio di 'allungare di qualche istante l'esistenza' di questa scrittrice su questo mondo e ringraziarla per l'operazione di analisi interiore che il lettore parallelamente fa, consciamente o inconsciamente durante il percorso di lettura. Inoltre, lo sperimentalismo continuo che ritroviamo nei suoi scritti, tanto nella lingua, quanto nello stile della stesura, nel contenuto e nel messaggio, va letto come un aspetto che l'ha contraddistinta e la contraddistingue nel contesto letterario del suo e del nostro tempo.

10 Concezione che troviamo nel poema Ode to a Grecian Urn di John Keats. 


\section{RIFERIMENTI BIBLIOGRAFICI}

Aiello, A., Di Maio, G., L'antigattopardo, Catania racconta Goliarda Sapienza, Associazione Documenta Catania DVD, 2012.

Andrigo, M., “L'evoluzione autobiografica di Goliarda Sapienza: stile e contenuti”, Providenti, G. (a cura di), Quel sogno d'essere di Goliarda Sapienza, Roma, Aracne, 2012, pp. 117-130.

Arena, M., "Il filo di mezzogiorno. Morte e rinascita attraverso la scrittura", Providenti, G. (a cura di), Quel sogno d'essere di Goliarda Sapienza, Roma, Aracne, 2012, pp. 149-155.

Bicchietti, G., “Esperienze dal carcere”, Providenti, G. (a cura di), Quel sogno d'essere di Goliarda Sapienza, Roma, Aracne, 2012, pp.181-190.

Cambria, A., Dopo l'Orca arriva la Gattoparda, "Il Giorno" 6 settembre 1979.

Hernández González, M.B., “La fortuna literaria de Goliarda Sapienza”, Arena Romanistica, 5 (2009), pp.140-152.

Langiano, A., "Lettera Aperta: il dovere di tornare”, Providenti, G., Quel sogno d'essere di Goliarda Sapienza, Roma, Aracne, 2012, pp.131-147.

Pellegrino, A., “Prefazione”, Sapienza, G., Il filo di mezzogiorno, Milano, Baldini\&Castoldi, 2015, pp. 5-12.

Providenti, G. (a cura di), Quel sogno d'essere di Goliarda Sapienza, Roma, Aracne, 2012.

----, La porta è aperta. Vita di Goliarda Sapienza, Catania, Villaggio Maori Edizioni, 2010.

Sapienza, G., Il filo di mezzogiorno, Milano, Baldini\&Castoldi, 2015.

----, L'Università di Rebibbia, Torino, Einaudi, 2012.

----, Le certezze del dubbio, Torino, Einaudi, 2013.

----, Lettera aperta, Palermo, Sellerio, 1997. 